\title{
Degradation of printing and dyeing wastewater by modified biochar catalyzed persulfate
}

\author{
Feng Ding ${ }^{1}$, Zichen $\mathrm{Luo}^{1}, \mathrm{Zi} \mathrm{xiao}^{1}$, Tengyan $\mathrm{Wu}^{1,2}$, and Hongbin Zhong ${ }^{1 *}$ \\ ${ }^{1}$ Hunan Provincial Key Laboratory of Fine Ceramics and Powder Materials, School of Materials and Environmental Engineering, Hunan \\ University of Humanities, Science and Technology, Loudi Hunan 417000, China \\ ${ }^{2}$ State Key Laboratory of Powder Metallurgy, Central South University, Changsha 410083, China.
}

\begin{abstract}
The biochar (BC) obtained from banana peel was used to catalyze persulfate (PS) to degrade the Direct Blue 86 (DB86) simulating and dyeing wastewater, which can obtain a much better degradation efficiency (71.1\% within 1440 min) compared with BC (insignificant) or PS (26.7\%) alone. Then BC was modified, and the influence of modified method, PS concentration, biochar content and temperature on DB86 degradation was also investigated. Results showed that the acid modified method of BC had advantage for the DB86 degradation, and 99\% decolorization efficiency can reach within $240 \mathrm{~min}$. The degradation of DB86 increased with PS concentration increasing, and then decreased slowly. Increasing the acid modified BC dosage and temperature can improve effectively the DB86 degradation. When the experimental conditions are PS $5 \times 10^{-3} \mathrm{M}$, acid modified BC $5.0 \mathrm{~g} / \mathrm{L}$, and $25^{\circ} \mathrm{C}$, the degradation of DB86 can reach $99 \%$ within $60 \mathrm{~min}$.
\end{abstract}

\section{Introduction}

With the development of society, the protection of environment is receiving more and more recognition. Dyeing and printing wastewater produced from dyeing and printing industry contains the classical kinds of pollutants with refractory organic compounds, which will cause serious pollution if they substandard discharge [1, 2]. Therefore, the treatment method of dyeing and printing wastewater degradation to reach the emission standard has caused the interests of scientists.

Advanced oxidation processes (AOPs) can degrade and mineralize organic pollutants efficiently, which have widely been applied in dyeing and printing wastewater treatment [3-6]. Among AOPs methods, persulfate oxidation process is considered as an environmental friendly oxidant, which has some advantages, such as prince moderate and easier to store and transport. Sulfate radicals $\left(\mathrm{SO}_{4}^{-}\right)$can be generated from persulfate oxidation process, which had similar high oxidation potential of $2.8 \mathrm{~V}$ but longer life span of 30-40 $\mu$ s than ${ }^{\circ} \mathrm{OH}[2,7]$. Metal ions are the mainly catalyst to introduce PS, however, the reuse and the toxicity of the metal catalysts, as well as the associated secondary contamination to the environment, also pose some challenges in such homogeneous oxidation processes.

Biochars, prepared by combusting biomass under low oxygen conditions, are attracting scientists' interests as promising environmental remediation materials [8]. Depending on pyrolysis conditions, biochar is of porous structure, and possesses large surface area [9] with a significant density of oxygen-containing functional groups, e.g. carboxyl (-COOH) and hydroxyl (-OH). Thus, biochar has been used as an adsorbent to removal organic contaminants $[10]$ and heavy metals $[11,12]$. In addition, biochar can be successfully utilized to disperse and stabilize nanoparticles to enhance their performance in environmental applications[7, 13, 14].

The research of contaminants adsorption and nanoparticles dispersion and stabilization about biochar had been systematically studied, however, there were not experiments about the catalytic performance of biochar. In this study, biochar was used to catalyze persulfate to degrade simulated dyeing and printing wastewater as the catalyst. Direct Blue 86 (DB86) was selected as the targeted pollutant. Moreover, the influence of modification method of biochar, biochars dosage, PS concentration, and temperature on the DB86 degradation was also examined. The point of zero charge $\left(\mathrm{pH}_{\mathrm{pzc}}\right)$ and, the Fourier transformed infrared spectrometer (FT-IR) of biochar and acid modified biochar were also investigated.

\section{Materials and Methods}

\subsection{Materials}

Direct Blue 86 (DB86, $\mathrm{C}_{32} \mathrm{H}_{14} \mathrm{CuN}_{8} \mathrm{Na}_{2} \mathrm{O}_{6} \mathrm{~S}_{2}$, CAS No. 1330-38-7) was obtained from Sigma (China). Sodium persulfate $\left(\mathrm{Na}_{2} \mathrm{~S}_{2} \mathrm{O}_{8}\right)$, Sulfuric acid $\left(\mathrm{H}_{2} \mathrm{SO}_{4}\right)$ and Sodium hydroxide $(\mathrm{NaOH})$ were purchased from Sinopharm Chemical Reagent CO., Ltd (China). All other reagents were analytical grade.

Biochars (BC) were produced by the pyrolysis of banana peels. The banana peels used in this work were

* Corresponding author: hbzhong@alum.imr.ac.cn 
collected locally in Loudi, Hunan Province, China and dried in oven at $80^{\circ} \mathrm{C}$. The dried banana peels were then calcinated in muffle furnace for $2 \mathrm{~h}$ at a temperature of $300^{\circ} \mathrm{C}$ to obtain BC. After cooling, $\mathrm{BC}$ were screened with a 60-mesh sieve, and then was used for the experiments. The biochars were treated with $1 \mathrm{~mol} / \mathrm{L} \mathrm{H}_{2} \mathrm{SO}_{4}$ or $\mathrm{NaOH}$ for $12 \mathrm{~h}$, then were washed with deionized water to natural $\mathrm{pH}$, dried at $80^{\circ} \mathrm{C}$ in the oven, the acid modified biochar (ACBC) or alkali modified biochar (ALBC) were obtained.

\section{2 persulfate oxidation procedure}

All reagents were prepared at an ambient temperature of $25 \pm 2^{\circ} \mathrm{C}$ using distilled water. Unless otherwise stated, the experimental procedures were as follows: $\mathrm{pH}$ of the DB86 dye solution (1 L) was natural pH. Specific dosages of BC, ACBC, or ALBC, and PS were added. The solution was then agitated at $800 \mathrm{rpm}$ for $30 \mathrm{~min}$ using a mechanical stirrer (Shin-Kwang, Taiwan). At selected time intervals, $10-\mathrm{mL}$ of the solution was taken from the reactor and filtered immediately through a $0.45 \mu \mathrm{m}$ membrane filter (Advantec, Japan). Residual DB86 concentration in the supernatant was determined following a procedure as described in section 2.3 .

The effect of ACBC dosage and PS concentration was also investigated under $0.5,1.0,2.0,3.0,4.0,5.0 \mathrm{~g} / \mathrm{L} \mathrm{Fe}^{0}$ dosages and $1 \times 10^{-3}, 2 \times 10^{-3}, 3 \times 10^{-3}, 5 \times 10^{-3}, 1 \times 10^{-2}, 2 \times 10^{-}$ ${ }^{2} \mathrm{~mol} / \mathrm{L}$ PS concentrations.

The effect of temperature on $\mathrm{PS} / \mathrm{Fe}^{0}$ process decolorization was investigated under the initial temperature conditions at $10,25,40$, and $55^{\circ} \mathrm{C}$ using a heating magnetic stirrer. All experiments were conducted in triplicate, and averaged values were used for data analysis.

\subsection{Analytical methods}

The DB86 concentration was analyzed using a spectrophotometer (Hach DR/3900, USA) at $618 \mathrm{~nm}$. Fourier transformed infrared spectrometer (FTIR) were used to characterize the unmodified biochar (BC) and acid modified biochar (ACBC). The functional groups and chemical bonds existing in prepared samples were identified on Nicolet iS10 spectra in the wavenumber range of $4000-400 \mathrm{~cm}^{-1}$. A pellet was created of $95 \% \mathrm{KBr}$ with $5 \%$ prepared nanoferrites for FTIR spectral analysis.

The point of zero charge $\left(\mathrm{pH}_{\mathrm{pzc}}\right)$ for $\mathrm{BC}$ and $\mathrm{ACBC}$ was determined according to the method reported by Srivastava et al., (2011) [15]. For determination of $\mathrm{pH}_{\mathrm{pzc}}$ for $\mathrm{BC}$ and $\mathrm{ACBC}$, a solution of $0.01 \mathrm{M} \mathrm{NaCl}, 50 \mathrm{~mL}$ was prepared, which $\mathrm{pH}$ was adjusted in the range between 2 and 12 by using $\mathrm{NaOH}$ or $\mathrm{HCl}$. Then the solution with different $\mathrm{pH}$ and $0.2 \mathrm{~g}$ of the $\mathrm{BC}$ and $\mathrm{ACBC}$ were added into flasks, and kept for $48 \mathrm{~h}$. After that, the $\mathrm{pH}$ of aolutions was measured. A praph was then plotted between $\mathrm{pH}_{\text {final }} \mathrm{vs} \mathrm{pH}_{\text {initial }}$. The point of intersection of the " $\mathrm{pH}_{\text {final }} \mathrm{Vs} \mathrm{pH}_{\text {initial }}$ " curves represents the $\mathrm{pH}_{\mathrm{pzc}}$ of the $\mathrm{BC}$ and $\mathrm{ACBC}$.

\section{Results and Disscussion}

\subsection{The efficiency of different processes on DB86 degradation}

Preliminary tests were carried out to assess the degradation power of each process, $\mathrm{PS}$ only, $\mathrm{BC}$ only, and $\mathrm{BC}$ activated $\mathrm{PS}(\mathrm{PS} / \mathrm{BC})$ processes. As shown in Fig. 1, process using $\mathrm{BC}$ only was inefficient for DB86 decolorization. Although PS is considered as a strong oxidant, it is difficult for PS to oxidize DB86 fastly, and the degradation of DB86 was $26.7 \%$ within $1440 \mathrm{~min}$. In comparison, the $\mathrm{BC}$ activated $\mathrm{PS}$ (PS/BC) process was capable of oxidizing the DB86, which achieved $71.1 \%$ degradation within $1440 \mathrm{~min}$, it is concluded that $\mathrm{BC}$ can efficiently activate PS to degrade DB86, the combination of $\mathrm{BC}$ and $\mathrm{PS}$ is a feasible and efficient approach to degrade DB86.

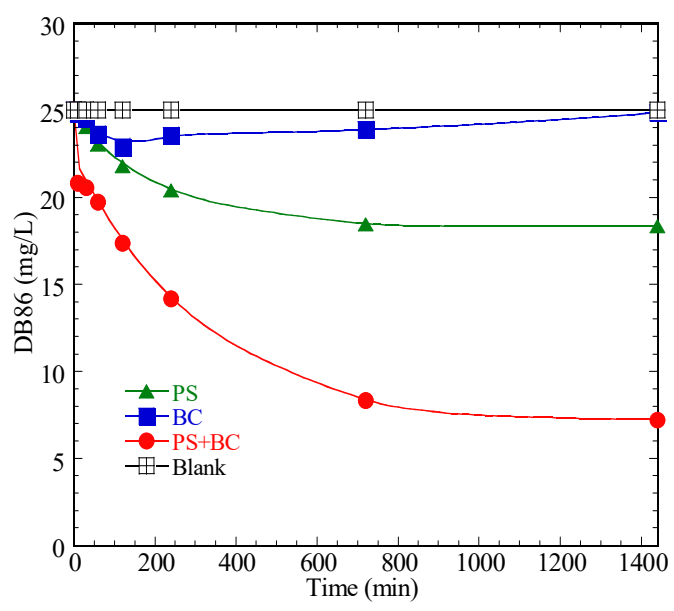

Fig. 1. Comparison of DB86 degradation in the different reaction systems. Experimental conditions: PS $5 \times 10^{-3} \mathrm{~mol} / \mathrm{L}$, $\mathrm{BC} 2.0 \mathrm{~g} / \mathrm{L}, 25^{\circ} \mathrm{C}$, natural $\mathrm{pH}$.

\section{2 different modified methods}

The different modified methods, including acid modified and alkaline modified methods, were used in this section, $\mathrm{ACBC}$ and $\mathrm{ALBC}$ were obtained. The influence of modified method of $\mathrm{BC}$ on the DB86 degradation was investigated, and results were shown in Fig. 2. The degradation of DB86 by ACBC activated persulfate (PS/ACBC) process was much higher than that of $\mathrm{BC}$ and ALBC. Alomstly 100\% degradation efficiency of DB86 can be obtained by PS/ACBC process within $240 \mathrm{~min}$ reaction time, in the mean while only $43.3 \%$ and $12.78 \%$ degradation efficiency obtained with $\mathrm{BC}$ and ALBC activated persulfate process. 


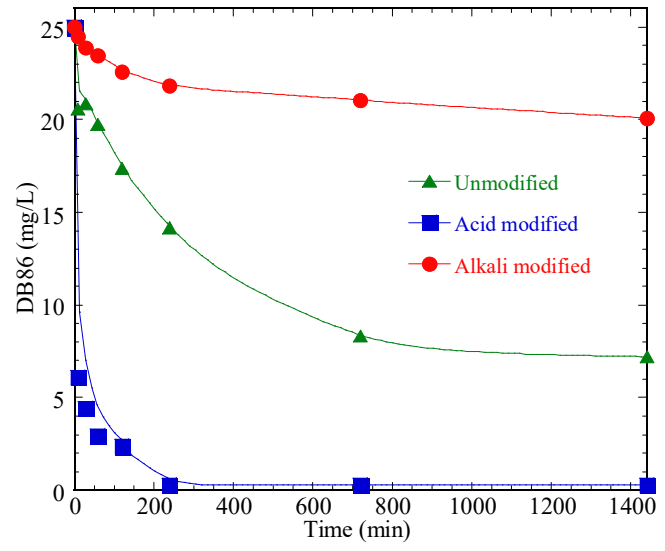

Fig. 2. Comparison of DB86 degradation in the different modified method of BC. Experimental conditions: PS $5 \times 10^{-3}$ $\mathrm{mol} / \mathrm{L}, \mathrm{BC}$ or $\mathrm{ACBC}$ or ALBC $2.0 \mathrm{~g} / \mathrm{L}, 25^{\circ} \mathrm{C}$, natural $\mathrm{pH}$.

The point of zero charge $\left(\mathrm{pH}_{\mathrm{pzc}}\right)$, reflecting acidic and basic groups on the surface of $\mathrm{ACBC}$, was determined to be 6.59 for $\mathrm{BC}$ and 2.48 for $\mathrm{ACBC}$ (Fig. 3). The variation in $\mathrm{pH}_{\mathrm{pzc}}$ indicated that the surface of $\mathrm{ACBC}$ carried more acidic groups. The acidic groups increasing may lead to the enhancement of catalytic property of ACBC.

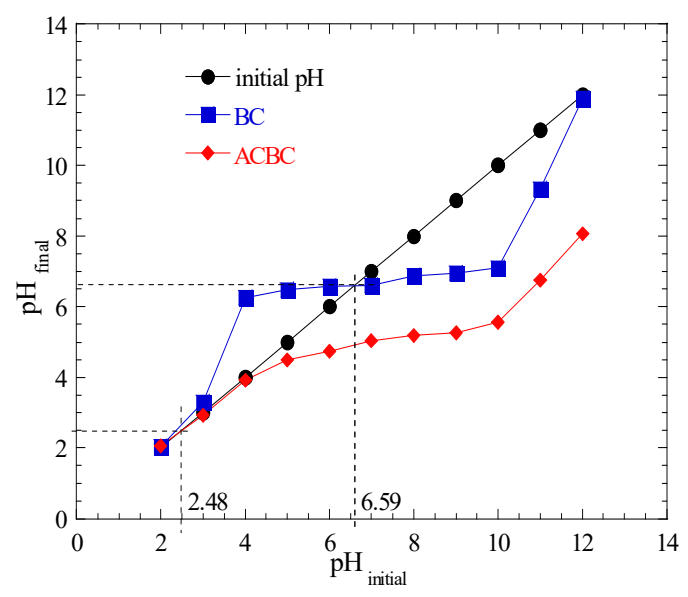

Fig. 3. $\mathrm{pH}_{\mathrm{pzc}}$ of $\mathrm{BC}$ and $\mathrm{ACBC}$.

The FT-IR spectra of $\mathrm{BC}$ and $\mathrm{ACBC}$ were shown in Fig.4. As observed, five distinct peaks appeared in the spectra, which are 3431, 2923, 2850, 1383, and $1064 \mathrm{~cm}^{-}$ ${ }^{1}$, respectively. The adsorption at $3431 \mathrm{~cm}^{-1}$ could be attributed to the $-\mathrm{OH}$ [7], the peaks at 2923 and $2850 \mathrm{~cm}^{-}$ ${ }^{1}$ were assigned to $\mathrm{C}-\mathrm{H}$ stretching vibrations of the $\mathrm{CH}_{3}$ and $\mathrm{CH}_{2}$ groups [16], the peak at $1383 \mathrm{~cm}^{-1}$ may be considered as the hydroxyl groups $\mathrm{C}=\mathrm{C}-\mathrm{H}$ [17] on the surface of BC. The peak at $1064 \mathrm{~cm}^{-1}$ should be attributed to $\mathrm{C}-\mathrm{O}-\mathrm{C}[18]$ carbon groups of $\mathrm{BC}$.

After the acid method was used, the ACBC was obtained. The intensity at $3431 \mathrm{~cm}^{-1}$ and $1383 \mathrm{~cm}^{-1}$ obviously increased compared with those peaks of $\mathrm{BC}$. Based on the observation, it is inferred that the $\mathrm{H}$-bonds with several $\mathrm{H}$-containing groups were formed on the surface of BC by using acid modified method, and therefore the intensity at $3431 \mathrm{~cm}^{-1}$ and $1383 \mathrm{~cm}^{-1}$ was increased. The degradation of DB86 by PS/ACBC process was much better than that of PS/BC, which just maybe due to the $\mathrm{H}$-containing groups increasing. It is also inferred that the $\mathrm{H}-$ containing groups may act as an activator for PS decomposition.

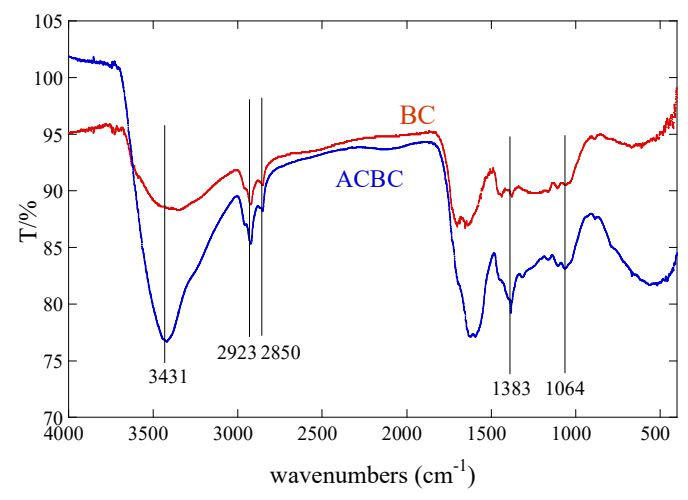

Fig. 4. FT-IR of BC and ACBC.

\subsection{Effect of ACBC dosage and PS concentration}

Fig.4a depicted the influence of $\mathrm{ACBC}$ dosages on the degradation of DB86 by ACBC-activated PS (PS/ACBC) process. The degradation of DB86 increased with the increasing of ACBC dosage in a range of $0.5-5.0 \mathrm{~g} / \mathrm{L}$. For example, approximately $77.8 \%$ of DB86 was obtained with $0.5 \mathrm{~g} / \mathrm{L}$ ACBC within $60 \mathrm{~min}$, whereas about $99 \%$ decomposition was achieved with $5.0 \mathrm{~g} / \mathrm{L}$ ACBC. This is because that a higher dosage of ACBC will provide more active sites for the activation of PS, which can accelerate the $\mathrm{SO}_{4}{ }^{-}$generation effectively.
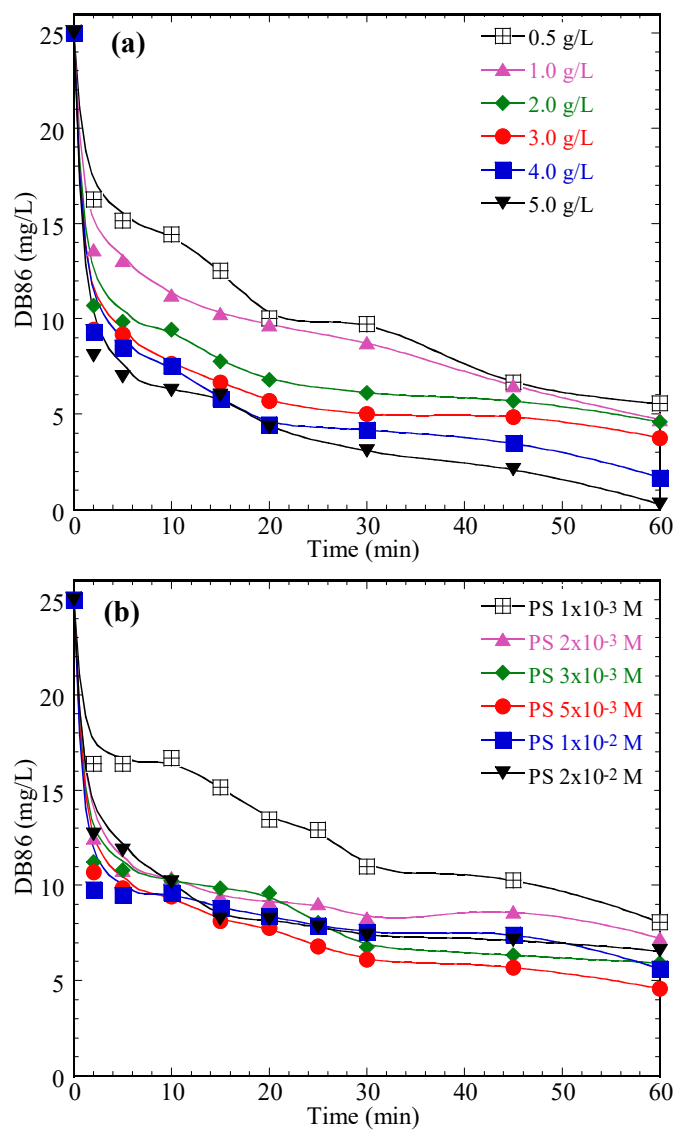

Fig. 5. Effect of ACBC dosage (a) and PS concentration (b) on degradation of DB86 by PS catalyzed with ACBC. 
Experimental conditions: PS $5 \times 10^{-3} \mathrm{~mol} / \mathrm{L}$ (a), ACBC $2.0 \mathrm{~g} / \mathrm{L}$ (b), natural $\mathrm{pH}$, and $25^{\circ} \mathrm{C}$.

The effect of PS dosage on DB86 degradation was also investigated. As shown in Fig. 4b, the degradation of DB86 was markedly improved with an increase of PS dosage ranging from $1 \times 10^{-3} \mathrm{~mol} / \mathrm{L}$ to $5 \times 10^{-3} \mathrm{~mol} / \mathrm{L}$. In the presence of $1 \times 10^{-3} \mathrm{~mol} / \mathrm{L}$ PS, the removal percentage of DB86 reached to approximately 67.8 within $60 \mathrm{~min}$. When the PS concentration enhanced to $5 \times 10^{-3} \mathrm{~mol} / \mathrm{L}$, the DB86 degradation also increased, and $81.7 \%$ of DB86 was achieved. As the source of $\mathrm{SO}_{4}{ }^{\cdot}$ radicals, more $\mathrm{SO}_{4}{ }^{-}$ - radicals was expected to be produced from higher PS concentration in the PS/ACBC system. However, the introduction of a higher PS concentration of $1 \times 10^{-2} \mathrm{~mol} / \mathrm{L}$ and $2 \times 10^{-2} \mathrm{~mol} / \mathrm{L}$ does not evince an obvious improvement in DB86 decolorization because of excess PS results in recombination of $\mathrm{SO}_{4}{ }^{-}$radicals into PS [19]. The scavenging effect of excess PS is expressed as Eqs. (1) and (2).

$$
\begin{gathered}
\mathrm{S}_{2} \mathrm{O}_{8}{ }^{2-}+\mathrm{SO}_{4}{ }^{\cdot-} \rightarrow \mathrm{S}_{2} \mathrm{O}_{8}{ }^{\cdot 2-}+2 \mathrm{SO}_{4}{ }^{2-} \\
\mathrm{SO}_{4}{ }^{-{ }^{--}}+\mathrm{SO}_{4}{ }^{\cdot-} \rightarrow \mathrm{S}_{2} \mathrm{O}_{8}{ }^{2-}
\end{gathered}
$$

The best decolorization of DB86 was achieved within $60 \mathrm{~min}$ in PS/ACBC process with a PS concentration of $5 \times 10^{-3} \mathrm{~mol} / \mathrm{L}$, which is a suitable PS concentration in this batch experiment.

\subsection{Effect of temperature on PS/ACBC decolorization of DB86}

The dyeing and printing processes are often operated at high temperature environment and therefore the dyeing and printing effluents are often produced at relatively high temperature [20]. The temperature is also has influence on PS oxidation process. Thus, the experiments were conducted with different temperatures $(10,25,40$, and $55^{\circ} \mathrm{C}$ ) to investigate the influence of temperature on the decolorization of DB86 by PS/ACBC process. Results were shown in Fig. 5. Temperature drastically affected DB86 depletion for the PS/ACBC systems. Higher temperature was more favorable for DB86 oxidation. The decolorization efficiency of DB86 was $67.2 \%$ within 60 min when the temperature was $10^{\circ} \mathrm{C}$. When the temperature was enhanced to $55^{\circ} \mathrm{C}$, almost $100 \%$ decolorization efficiency can be obtained.

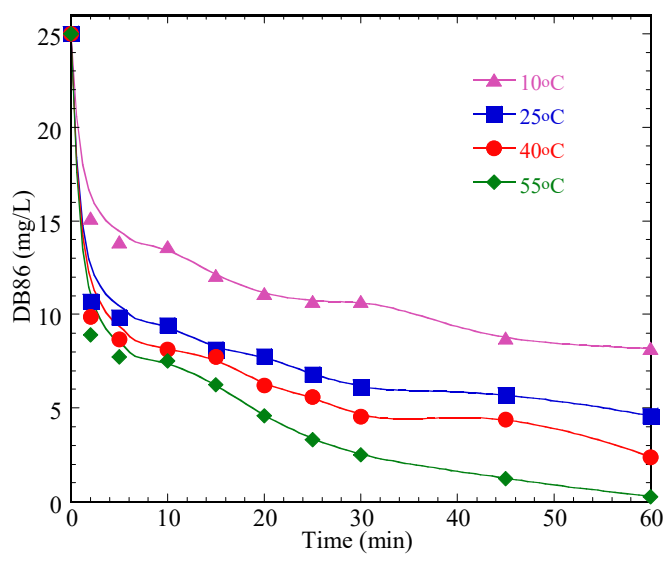

Fig. 6. Effect of temperature on degradation of DB86 by PS catalyzed with ACBC. Experimental conditions: PS $5 \times 10^{-3}$ $\mathrm{mol} / \mathrm{L}, \mathrm{ACBC} 2.0 \mathrm{~g} / \mathrm{L}$, and natural $\mathrm{pH}$.

The decolorization of DB86 by PS/ACBC process was essentially limited by the rate of $\mathrm{SO}_{4} \cdot$ generation. Heat can activate $\mathrm{PS}$ to generated $\mathrm{SO}_{4}{ }^{-}$, which can significantly increase the generation rate of $\mathrm{SO}_{4}{ }^{-}$radicals. In addition, high temperature can accelerat the molecular motion, which can enhance the contaction rate between PS and ACBC. Therefore, high temeprature would obtain a higher decolorization efficiency of DB86 by PS/ACBC system.

\subsection{Reusability test of $B C$ aggregate}

It is expected that the temperature of the fresh textile dyeing wastewaters in the effluent is usually high. Considering the practical application of PS/ACBC in-situ for treating fresh dyeing effluent, the reusability of $\mathrm{ACBC}$ aggregate in the PS/ACBC system for DB86 decolorization was conducted at $55^{\circ} \mathrm{C}$. Fig. 7 showed the degradation of DB 86 decreased gradually from $99 \%$ in the $1^{\text {st }}$ cycle within $60 \mathrm{~min}$ to $72.02 \%$ after the $3^{\text {rd }}$ cycle. The catalytic effect of ACBC decreased gradually with the increase of cycling times. The reason may be that with the increasing of cycling times, more and more holes were blocked in the layered structure of ACBC, which leaded to the activation sites reduced. Thus, the degradation of DB86 decreased.

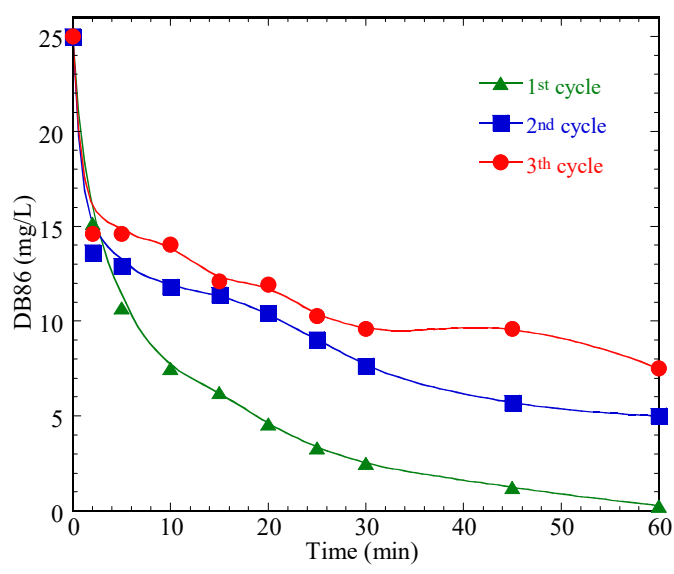

Fig. 7. Reusability test on DB86 decolorization in the PS/ACBC system. Experiment condition for all cycles: PS $5 \times 10^{-3} \mathrm{~mol} / \mathrm{L}, 55^{\circ} \mathrm{C}$, and natural $\mathrm{pH}$.

\section{Conclusions}

In this study, the degradation of DB86 by BC-activated PS was investigated. $\mathrm{BC}$ can effectively activate persulfate to degrade DB86, and the degradation of DB86 can be further enhanced after the $\mathrm{BC}$ was modified by sulfate acid. The pHpzc and FT-IR indicated the acid modified method can enhance the $\mathrm{H}$-bonds and $\mathrm{H}$-containing groups on the $\mathrm{BC}$ surface, which would increase the catalytic performance of $\mathrm{BC}$. The degradation efficiency of DB86 increased with the increase of ACBC, PS dosage and temperature. The reuse performance of $\mathrm{ACBC}$ was further studied, which showed the catalytic property of 
$\mathrm{ACBC}$ decreased with the increasing of reuse times. The results demonstrated that the better catalytic performance of BC can be obtained by using the acid modified method, which will accelerate the dye wastewater degradation by activated persulfate.

This work is supported by the planned Science and Technology project of Hunan Province, China (2016TP1028, 16C0826, 2017JJ3125) and supported by the double first-class discipline construction program of Hunan province (16B136).

\section{References}

1. F. Ding, H. Chen, S. Zhang, T. Zhao, N. Liu, J. Environ. Manag. 200 (2017) 325-334.

2. X. Lin, Y. Ma, J. Wan, Y. Wang, Y. Li, Chemosphere, 214 (2019) 642-650.

3. J.E. Grebel, J.J. Pignatello, W.A. Mitch, Environ. Sci. Technol. 44 (2010) 6822.

4. Y. Guo, Q. Xue, H. Zhang, N. Wang, S. Chang, H. Wang, H. Pang, H. Chen, Rsc Adv. 8 (2017) 80-90.

5. C.-H. Liao, S.-F. Kang, F.-A. Wu, chemosphere, 44 (2001) 1193-1200.

6. M.-C. Lu, Y.-F. Chang, I.M. Chen, Y.-Y. Huang, J. Environ. Manag. 75 (2005) 177-182.

7. I. Hussain, M. Li, Y. Zhang, Y. Li, S. Huang, X. Du, G. Liu, W. Hayat, N. Anwar, Chem. Eng. J. 311 (2017) 163-172.

8. Y. Wu, J. Guo, Y. Han, J. Zhu, L. Zhou, Y. Lan, Chemosphere, 200 (2018) 373-379.
9. N. Liu, A.B. Charrua, C.H. Weng, X. Yuan, F. Ding, Bioresour. Technol. 198 (2015) 55-62.

10. J.-K. Im, L.K. Boateng, J.R.V. Flora, N. Her, K.-D. Zoh, A. Son, Y. Yoon, Sep. Purif. Technol. 123 (2014) 96-105.

11. P. Liu, C.J. Ptacek, K.M.A. Elena, D.W. Blowes, W.D. Gould, Y.Z. Finfrock, A.O. Wang, R.C. Landis, J. Hazard. Mater. 347 (2018) 114-122.

12. T.M. Abdel-Fattah, M.E. Mahmoud, S.B. Ahmed, M.D. Huff, J.W. Lee, S. Kumar, J. Ind. Eng. Chem. 22 (2015) 103-109.

13. A. Khataee, B. Kayan, P. Gholami, D. Kalderis, S. Akay, Ultrason. Sonochem. 39 (2017) 120-128.

14. A. Khataee, B. Kayan, P. Gholami, D. Kalderis, S. Akay, L. Dinpazhoh, Ultrason. Sonochem. 39 (2017) 540-549.

15. V. Srivastava, C.H. Weng, V.K. Singh, Y.C. Sharma, J. Chem. Eng. Data, 56 (2011) 1414-1422.

16. H. Zhang, G. Xue, H. Chen, X. Li, Chemosphere, 191 (2018) 64-71.

17. J.H. Yuan, R.-K. Xu, H. Zhang, Bioresour. Technol. 102 (2011) 3488-3497.

18. I. Hussain, M. Li, Y. Zhang, Y. Li, S. Huang, X. Du, G. Liu, W. Hayat, N. Anwar, Chem. Eng. J. (2016) S1385894716316473.

19. C.-H. Weng, F. Ding, Y.-T. Lin, N. Liu, Sep. Purif. Technol. 147 (2015) 147-155.

20. N. Liu, F. Ding, C.-H. Weng, C.-C. Hwang, Y.-T. Lin, Sep. Purif. Technol. 169 (2016) 230-240. 